\title{
Note on Coulson et al
}

IIALIV F. SEGAL, Institute for Child and Family Development. The University of North Carolina at Greensboro, Greensboro, N.C. 27412

Most researchers use the term DRL to describe a set of reinforcement contingencies that shape spaced responding. On a DRL $x$ second reinforcement schedule, every response that is spaced at least $\mathrm{x}$ seconds from the preceding response is reinforced as soon as it occurs. A response, $\mathrm{n}$, that occurs earlier than $\mathrm{x}$ seconds after the preceding response. $\mathrm{n}-1$, is not reinforced, and moreover resets the reinforcement timer so that the next response, $n+1$, will only be reinforced if it occurs at least $x$ seconds after its preceding response, $n$.

As I understand the description of the procedure in the Coulson, Gardner, \& Coulson (1969) experiment, a response was reinforced with a delay of $x$ seconds. That is, $x$ seconds after the response, $n$, occurred, a reinforcer was delivered, unless additional responses, $n+i$, occurred during the delay interval. In that case, reinforcement was postponed to $\mathrm{x}$ seconds after the last, $n+\mathrm{ith}^{\mathrm{th}}$, response. In the commonly accepted terminology of reinforcement schedules, this procedure would be described as a Tandem FR 1 DRO $x$, indicating that two contingencies had to be met in succession: first, a response had to be emitted, and then $x$ seconds had to pass without a response (DRO: differential reinforcement of other behavior), in order for a reinforcer to be delivered.

Terminology is, of course, arbitrary, and there is no reason why definitions of terms cannot change to suit changing needs of the science. However, communication is facilitated where consistent usage obtains, and where novel usages are clearly announced and justified. The novelty of the usage of DRL in this paper may not have been apparent to the authors. It does not, in any case, appear justified, inasmuch as there is another term to describe the procedure, viz, Tandem FR 1 DRO $x$, and confusion between the DRL and Tandem FR 1 DRO procedure is not desirable.

\section{REFERENCE}

COULSON, G., GARDNER, L., \& COULSON, V. Acquisition of lever pressing in rats under DRL contingencies. Psychonomic Science, 1969, 17 . 265-266.

\section{Reply to Segal}

G. COULSON, L. GARDNER, and $V$. COULSON, York University, Downsview, Ont., Canada

As Segal (1970) points out, our use of DRL to describe the basic contingency in our experiment is incorrect in the light of conventional and long-established usage. In contrast to the DRL contingency normally used, where wait-respond is reinforced, our schedule reinforced the respond-wait chain. In terms of effect, considering response rate, both of these schedules reinforce low rates.
We suggest that DRL be used as a generic term to describe that class of schedules whose common element is the positive reinforcement of low rates. This usage would preserve the notion that the schedules in that class reinforce, and consequently produce, low rates of responding as implied by the phrase abbreviated as DRL. The usual DRL schedule and the schedule used in our experiment are both members of this class. We suggest that they be differentiated by labels like delayed-response DRL and delayed-reinforcement DRL, respectively.
We feel, then, that our error was not in using DRL to refer to the schedule we employed but in using the term without specific modification. To use Segal's phrase, we failed to "announce and justify" our novel usage. We hope this note clarifies our position. We thank Segal for bringing the ambiguity to our attention and thereby providing us with the opportunity to clarify our novel use of an established term.

REFERENCF

SEGAL. E. Comment on Coulson et al Psychonomic Science, 1970. 19.9. 